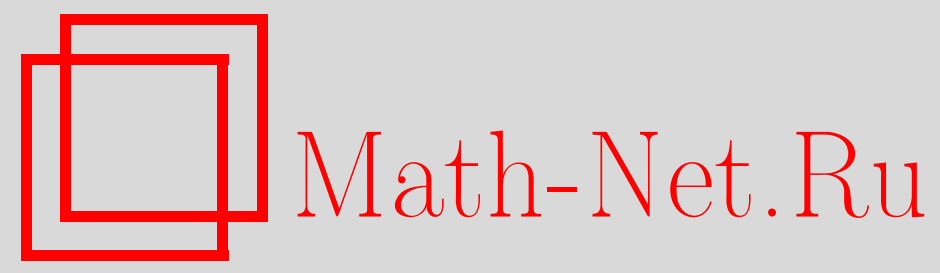

А. В. Нагаев, Крамеровские большие уклонения в случае, когда крайнее сопряженное распределение имеет тяжелый хвост, Теория вероятн. и ее примен., 1998, том 43, выпуск $3,456-475$

DOI: https://doi.org/10.4213/tvp1554

Использование Общероссийского математического портала Math-Net.Ru подразумевает, что вы прочитали и согласны с пользовательским соглашением http://www . mathnet.ru/rus/agreement

Параметры загрузки:

IP : 54.147 .182 .235

26 апреля 2023 г., 05:13:53

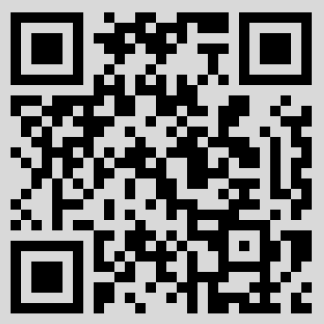




\title{
КРАМЕРОВСКИЕ БОЛЬШИЕ УКЛОНЕНИЯ В СЛУЧАЕ, КОГДА КРАЙНЕЕ СОПРЯЖЕННОЕ РАСПРЕДЕЛЕНИЕ ИМЕЕТ ТЯЖЕЛЫЙ ХВОСТ ${ }^{1)}$
}

\begin{abstract}
Рассматривается классическая проблема больших уклонений в предположении, что одностороннее условие Крамера выполнено в замкнутом промежутке. Предполагается, что крайнее сопряженное распределение принадлежит области притяжения устойчивого закона. Устанавливается граница, до которой асимпітотическое представление Крамера-Петрова сохраняет силу.
\end{abstract}

Ключевые слова и фразы: сопряженное распределение, медленно изменяющаяся функция, монотонная $\varepsilon$-аппроксимация.

\section{1. Введение и основные результаты}

В настоящее время теория болыших уклонений получила достаточное развитие и находит применение во многих разделах теории вероятностей. Ее классическая часть имела дело с вероятностями больших уклонений сумм независимых случайных величин. В последующем она охватила гораздо более общие схемы. В наши планы, однако, не входит обзор этой теории. Желающие получить о ней более полное представление могут обратиться к работам [1]-[6].

Целью данной статьи является решение одной задачи, связанной с классическим результатом Крамера [7]. Приводимые ниже результаты были анонсированы в [8].

Пусть случайные величины $\xi, \xi_{1}, \xi_{2}, \ldots$ независимы и имеют общее распределение $F(x)$. Положим

$$
\zeta_{n}=\xi_{1}+\cdots+\xi_{n}
$$

и

$$
F_{n}(x)=\mathbf{P}\left\{\zeta_{n}<x\right\}, \quad P_{n}(x)=\mathbf{P}\left\{\zeta_{n} \geqslant x\right\} .
$$

Предположим, что выполнено одностороннее условие Крамера, т.е.

$$
0=\inf \left(s: \mathbf{E} e^{s \xi}<\infty\right)<\sup \left(s: \mathbf{E} e^{s \xi}<\infty\right)=\lambda<\infty
$$

*Университет Н. Коперника, Торунь, Польша.

1) При поддержке KBN, грант РВ 591/P03/95/08. 
Условие (1) означает, что $P_{1}(x)$, правый хвост исходного распределения $F(x)$, показательно быстро убывает при $x \rightarrow \infty$. Через $f(s)$ обозначим производящую функцию $\xi$. Согласно (1) $f(s)<\infty$, по меньшей мере, для $s \in(0, \lambda)$. Положим

$$
M(s)=\frac{f^{\prime}(s)}{f(s)}, \quad M=\lim _{s \rightarrow \lambda} M(s)
$$

Поскольку

$$
M^{\prime}(s)=(f(s))^{-1} \int_{-\infty}^{\infty}(x-M(s))^{2} e^{s x} d F(x)>0,
$$

уравнение $x=M(s)$ имеет единственный корень при $M(0)<x<M$. Следует заметить, что $M(0)$ или конечно или равно $-\infty$.

Определим функцию уклонений

$$
\rho(x)=\inf _{0 \leqslant s<\lambda} f(s) e^{-s x} .
$$

Легко видеть, что при $M(0)<x<M$

$$
\rho(x)=f(s(x)) \exp (-x s(x)) .
$$

Далее положим

$$
M_{\delta}= \begin{cases}M-\delta, & \text { если } M<\infty \\ \delta^{-1}, & \text { если } M=\infty\end{cases}
$$

и

$$
m_{\delta}= \begin{cases}M(0)+\delta, & \text { если } M(0) \text { конечно, } \\ -\delta^{-1}, & \text { если } M(0)=-\infty\end{cases}
$$

С этого момента $\delta$ будет обозначать произвольно малое фиксированное число, так что в (4) и (5) значения $\delta$ могут быть разными.

Следующая теорема хорошо известна (см. [9], ср. с [19]).

Теорема 1. Пусть выполнено условие (1). Тогда

$1^{\circ}$. Если $F(x)$ нерешетчато, то при $n \rightarrow \infty$

$$
P_{n}(x)=A\left(x n^{-1}\right) n^{-1 / 2} \rho^{n}\left(x n^{-1}\right)(1+o(1))
$$

равномерно по $x, m_{\delta} \leqslant x n^{-1} \leqslant M_{\delta}$;

$2^{\circ}$. Если $\xi_{1}$ принимает значения $a+k h, k=0, \pm 1, \pm 2, \ldots$, где $h$ является максимальным шагом распределения, то

$$
P_{n}(x)=B\left(x n^{-1}\right) n^{-1 / 2} \rho^{n}\left(x n^{-1}\right)(1+o(1))
$$


равномерно по $x, x=n a+k h, m_{\delta} \leqslant x n^{-1} \leqslant M_{\delta}$. Здесь

$$
\begin{aligned}
& A(x)=\left(2 \pi s^{2}(x) M^{\prime}(s(x))\right)^{-1 / 2} \\
& B(x)=\left(2 \pi M^{\prime}(s(x))\right)^{-1 / 2} \frac{h}{1-e^{-s(x) h}}
\end{aligned}
$$

a $s(x)$ корень уравнения $M(s)=x$ или, иначе, $s(x)=M^{-1}(x)$.

В связи с теоремой возникает ряд вопросов. В частности,

1) Насколько можно расширить область уклонений, сохраняя справедливость асимптотических соотношений (6) и (7)?

2) Какая асимптотика возникает, когда $x n^{-1}<m_{\delta}$ или $x n^{-1}>M_{\delta}$, а соотношения (6) и (7) не выполняются?

3) Какая асимптотика ожидается при $\lambda=\infty$ ?

Случай $x n^{-1}<m_{\delta}$ изучался в [10] и [11]. В данной работе нас интересует случай $x n^{-1}>M_{\delta}$.

Легко предвидеть, что ширина области больших уклонений, допускающей крамеровскую асимптотику, зависит от регулярности изменения $P_{1}(x)$. Некоторые условия регулярности, гарантирующие указанную асимптотику для всех $x \geqslant n m_{\delta}$, были предложены в [12]. Они относятся к случаю $\lambda=\infty$. Любопытные явления, возникаюшие, когда $\xi$ целочисленна, описаны в [13].

Если же $\lambda<\infty$, то нам приходится различать случаи $f(\lambda)<\infty$ и $f(\lambda)=\infty$. В этой работе мы сосредоточимся на первом из этих случаев. Второму случаю будет посвящена отдельная работа.

Если $f(\lambda)<\infty$, то существует крайнее правое сопряженное распределение

$$
H(x)=\frac{1}{f(\lambda)} \int_{-\infty}^{x} e^{\lambda u} d F(u) .
$$

Из (1) следует, что левый хвост $H(x)$ убывает экспоненциально быстро. Очевидно, (см. (2) и следующее за ним соотношение) $M=M(\lambda)$ и $M^{\prime}(\lambda)$ являются соответственно математическим ожиданием и дисперсией распределения $H(x)$. Если $M^{\prime}(\lambda)<\infty$, то легко показать, что асимптотики (6) и (7) могут быть продолжены до области $x n^{-1} \in\left(m_{\delta}, M+X \sqrt{M^{\prime}(\lambda)}\right)$ для любого фиксированного $X>0$. Так что остается рассмотреть случай, когда $H(x)$ имеет тяжелый правый хвост.

В дальнейшем мы предполагаем, что для $x \geqslant 0$

$$
1-H(x)=x^{-\alpha} m(x)
$$

где $0<\alpha \leqslant 2$, а $m(x)$ медленно изменяется при $x \rightarrow \infty$. Более того, мы предполагаем, что $M^{\prime}(\lambda)=\infty$, т.е. при $\alpha=2$

$$
\int_{1}^{\infty} u^{-1} m(u) d u=\infty
$$


Пусть

$$
b_{n}= \begin{cases}\inf (x: n(1-H(x)) \leqslant 1), & \text { если } 0<\alpha<2, \\ \inf \left(x: n x^{-2} \int_{-x}^{x} u^{2} d H(u) \leqslant 1\right), & \text { если } \alpha=2,\end{cases}
$$

и

$$
a_{n}= \begin{cases}0, & \text { если } 0<\alpha<1, \\ n \int_{-b_{n}}^{b_{n}} u d H(u), & \text { если } \alpha=1, \\ n M, & \text { если } 1<\alpha \leqslant 2 .\end{cases}
$$

Обозначим через $H_{n}(x) n$-ю свертку (8). Хорошо известно, что (см., например, гл. II в [14]), при выполнении (9) и $n \rightarrow \infty$

$$
\sup _{x}\left|H_{n}\left(x b_{n}+a_{n}\right)-\int_{\infty}^{x} g_{\alpha}(u) d u\right|=o(1),
$$

где $g_{\alpha}(x)$ - плотность спектрально положительного устойчивого закона с характеристическим показателем $\alpha$.

Далее, положим

$$
x_{n}= \begin{cases}\epsilon_{n} b_{n}, & \text { если } 0<\alpha<1, \\ a_{n}-z_{n} b_{n}, & \text { если } 1 \leqslant \alpha \leqslant 2,\end{cases}
$$

где $\epsilon_{n} \rightarrow 0, \epsilon_{n} b_{n} / n \rightarrow \infty$, а $z_{n} \rightarrow \infty, z_{n}=o\left(a_{n} / b_{n}\right)$. Заметим, что $x_{n}>n M_{\delta}$ изменяется в области больших уклонений для $H_{n}(x)$.

Без потери общности в дальнейшем мы предположим, что случайная величина $\xi$ либо нерешетчата, либо целочисленна.

Теорема 2. Если выполнено (9), то утверждение теоремы 1 сохраняет силу для $n m_{\delta} \leqslant x \leqslant x_{n}$. Если же $x-a_{n} \asymp b_{n}$, то

$$
P_{n}(x)=\Delta(\lambda) b_{n}^{-1} g_{\alpha}\left(b_{n}^{-1}\left(x-a_{n}\right)\right) f^{n}(\lambda) e^{-\lambda x}(1+o(1)),
$$

ade

$$
\Delta(\lambda)= \begin{cases}\lambda^{-1}, & \text { если } \xi \text { нерешетчата }, \\ \left(1-e^{-\lambda}\right)^{-1}, & \text { если } \xi \text { челочисленна. }\end{cases}
$$

Таким образом, $x_{n}$ является гранипей, до которой может быть продолжена асимптотика (6). Случай, когда $b_{n}^{-1}\left(x-a_{n}\right) \longrightarrow \infty$, заслуживает специального исследования.

\section{2. Предварительные замечания}

Пусть для любого $s \in(0, \lambda]$ случайные величины $\xi(s), \xi_{1}(s), \xi_{2}(s), \ldots$ независимы и имеют общее распределение

$$
F_{s}(x)=\frac{1}{f(s)} \int_{-\infty}^{x} e^{s u} d F(u)
$$


называемое сопряженным по отношению к $F(u)$. Очевидно, что

$$
\mathbb{E} \xi(s)=M(s), \quad \mathbf{D} \xi(s)=M^{\prime}(s) .
$$

Напомним, что в рассматриваемом случае существует крайнее правое сопряженное распределение $F_{\lambda}(x)=H(x)$ (см. (8)). При этом для любого фиксированного $s \in(0, \lambda)$ распределение $F_{s}(x)$ принадлежит области нормального притяжения нормального закона, тогда как $H(x)$ лежит в области притяжения устойчивого закона с бесконечной дисперсией.

Положим

$$
\zeta_{n}(s)=\xi_{1}(s)+\cdots+\xi_{n}(s)
$$

и рассмотрим меры

$$
Q_{n s}(A)=\sqrt{2 \pi n M^{\prime}(s)} \mathbf{P}\left\{\zeta_{n}(s)-n M(s) \in A\right\} .
$$

Если $\xi$ нерешетчата, то из [15] следует, что $Q_{n s}$ слабо сходится к мере Лебега равномерно по $s \in\left[s_{1}, s_{2}\right] \subset(0, \lambda)$, т.е. для любой функции $g(x)$, имеюшей компактный носитель,

$$
\int g(x) Q_{n s}(d x) \rightarrow \int g(x) d x
$$

равномерно по $s$.

Легко видеть, что равномерная сходимость сохранится, если предположить, что $g(x)$ принадлежит некоторому классу равностепенно непрерывных функций.

Поэтому теорема 1 немедленно следует из хорошо известного представления

$$
\mathbf{P}\left\{\zeta_{n}>x\right\}=\frac{\rho^{n}\left(x n^{-1}\right)}{\sqrt{2 \pi n M^{\prime}\left(s\left(x n^{-1}\right)\right)}} \int_{0}^{\infty} e^{-s u} Q_{n s}(d u)
$$

Если $M^{\prime}(\lambda)<\infty$, то $Q_{n s}$ сходится равномерно по $s \in\left[s_{1}, \lambda\right]$. Если же $M^{\prime}(\lambda)=\infty$, то приходится искать такую пороговую последовательность $s_{n} \rightarrow \lambda$, что указанная сходимость выполняется равномерно по $s \in\left[s_{1}, s_{n}\right]$. Ниже будет показано, что $s_{n}$ находится из уравнения $n^{-1} x_{n}=M(s)$, где $x_{n}$ определено соотношениями (12).

Легко предвидеть, что все сведется к предельной задаче для схемы серий. В зависимости от величины $\alpha$ в формуле (9) приходится рассматривать пять случаев. Если случаи $0<\alpha<1$ и $0<\alpha<2$ довольно просты, то пограничные случаи

$$
\alpha=1, \int x d H(x)=\infty \text { или } \int x d H(x)<\infty
$$

и $\alpha=2$ требуют некоторых усилий. 
Стоит заметить, что (9) выполняется, в частности, если для $x>$ $x_{0}>0$

$$
P_{1}(x)=e^{-\lambda x} x^{-\alpha-1} l(x),
$$

где $l(x)$ медленно изменяется. Интегрируя по частям и используя теорему VIII.9.1 из [16], получаем, что

$$
1-H(x) \sim \frac{\lambda}{\alpha f(\lambda)} x^{-\alpha} l(x),
$$

T.e.

$$
m(x) \sim \frac{\lambda}{\alpha f(\lambda)} l(x) .
$$

Однако целочисленная случайная величина $\xi$ не может удовлетворять соотношению (14) для всех $x \geqslant x_{0}$. Если же предположить, что (14) выполнено только для целых $x$, то

$$
\mathrm{P}\{\xi=k\} \sim\left(1-e^{-\lambda}\right) e^{-\lambda k} k^{-\alpha-1} l(k),
$$

т.е. интегральная и локальная формы условия совпадают. При этом ни (14), ни (15) не влекут за собой (9) без дополнительных ограничений на $m(x)$.

Ниже приведено доказательство только для нерешетчатой случайной величины $\xi$. Изменения, необходимые в случае целочисленной $\xi$, очевидны.

\section{3. Замечания по поводу медленно изменяющихся функций}

Условимся обозначать буквой $c$ любую положительную постоянную, т.е. величину, независящую от $n$ и $x$. Буквой $\theta$ мы обозначим любую величину, принимающую значения из отрезка $[-1,1]$, и пусть $\omega(t)$ означает любую функцию такую, что $\lim _{t \rightarrow \infty} \omega(t)=0$. При таком соглашении, например, $c+c=c, c^{2}=c, \omega(t)+\omega(t)=\omega(t)$ и т.д. чTO

Пусть $m(x)>0$ и медленно изменяется при $x \rightarrow \infty$. Предположим,

$$
\widehat{m}(x)=\int_{0}^{x} u^{-1} m(u) d u
$$

конечна для всех $x>0$. Хорошо известно (см., например, теорему VIII.9.1 в [16]), что если

$$
\int_{0}^{\infty} u^{-1} m(u) d u=\infty
$$

то $\widehat{m}(x)$ медленно изменяется и

$$
m(x)=o(\widehat{m}(x))
$$


Если же

$$
\int_{0}^{\infty} u^{-1} m(u) d u<\infty
$$

To

$$
\tilde{m}(x)=\int_{x}^{\infty} u^{-1} m(u) d u
$$

медленно изменяется и

$$
m(x)=o(\tilde{m}(x))
$$

C помошью хорошо известного представления Караматы (см., например, следствие VIII.9 в [16]) можно утверждать, что существует такое $X>0$, что для $\min (x, y)>X$ и любого $\delta>0$

$$
\frac{1}{2} \min \left(\left(\frac{x}{y}\right)^{\delta},\left(\frac{y}{x}\right)^{\delta}\right) \leqslant \frac{m(x)}{m(y)} \leqslant 2 \max \left(\left(\frac{x}{y}\right)^{\delta},\left(\frac{y}{x}\right)^{\delta}\right) .
$$

Следующее свойство удобно сформулировать в виде леммы.

Лемма 1. Пусть $m(x)$ медленно изменяется при $x \rightarrow \infty$, а $g(x)$ удовлетворяет следующим условиям:

$1^{\circ}$. $\partial \Omega A$ любoro $\epsilon>0$

$$
\int_{\epsilon}^{1 / \epsilon}|g(u)| d u<\infty
$$

$2^{\circ}$. существует $\delta>0$ maкое, что $\partial \Omega я$ некоторого $\beta$

$$
\int_{0}^{\epsilon}|u|^{\beta-\delta}|g(u)| d u<\infty \quad u \quad \int_{1 / \epsilon}^{\infty}|u|^{\beta+\delta}|g(u)| d u<\infty
$$

$3^{\circ} . \partial \Omega s \tau \rightarrow 0$

$$
\int_{0}^{\tau} u^{\beta}|g(u)| d u=o\left(m\left(\tau^{-1}\right)\right)
$$

Tогдa $n p u \tau \rightarrow 0$

$$
I(\tau)=\int_{0}^{\infty} u^{\beta} m(u) g(u \tau) d u \sim \tau^{-(\beta+1)} m\left(\tau^{-1}\right) \int_{0}^{\infty} u^{\beta} g(u) d u .
$$

Мы опускаем доказательство этой леммы, которое мало отличается от доказательства приведенных свойств функций с медленным изменением.

\section{4. Свойства производящей функции}

Положим

$$
h(\tau)=\int_{-\infty}^{\infty} e^{-\tau u} d H(u)
$$


Ясно, что

$$
h(\tau)=\frac{f(\lambda-\tau)}{f(\lambda)}
$$

и что $h(\tau)$ аналитична в полосе $0<\operatorname{Re} \tau<\lambda$.

Обозначим через $l(x)$ любую функцию с медленным изменением такую, что

$$
\lim _{x \rightarrow \infty} \frac{l(x)}{m(x)}=c>0 .
$$

Следует подчеркнуть, что конкретное значение $c$ не играет роли.

Лемма 2. В зависимости от $\alpha$, функция $1-h(\tau)$ допускает одно из следующих представлений:

$$
1-h(\tau)= \begin{cases}\tau^{\alpha} l\left(\tau^{-1}\right), & \text { eсли } 0<\alpha<1, \\ \tau\left(\hat{m}\left(\tau^{-1}\right)-l\left(\tau^{-1}\right)\right), & \text { ecлu } \alpha=1, h^{\prime}(0)=-\infty \\ -h^{\prime}(0) \tau-\tau\left(\tilde{m}\left(\tau^{-1}\right)+l\left(\tau^{-1}\right)\right), & \text { ecлu } \alpha=1, h^{\prime}(0)>-\infty \\ -h^{\prime}(0) \tau-\tau^{\alpha} l\left(\tau^{-1}\right), & \text { еслu } 1<\alpha<2 \\ -h^{\prime}(0) \tau-\tau^{2}\left(\hat{m}\left(\tau^{-1}\right)+l\left(\tau^{-1}\right)\right), & \text { еслu } \alpha=2 .\end{cases}
$$

Здесь, как $u$ в (16) $u(17)$,

$$
\widehat{m}(x)=\int_{0}^{x} u^{-1} m(u) d u, \quad \tilde{m}(x)=\int_{x}^{\infty} u^{-1} m(u) d u .
$$

I о каз а т л в с т о. Интегрируя по частям, получаем

$$
h(\tau)=\tau \int_{-\infty}^{\infty} e^{-\tau u} H(u) d u=1-\tau \int_{0}^{\infty} e^{-\tau u}(1-H(u)) d u+O(\tau)
$$

Напомним, что $H(-u)=O\left(e^{-\lambda u}\right)$ при $u \rightarrow \infty$.

Подобным образом, если $h^{\prime}(0)>-\infty$, то

$$
\begin{aligned}
h(\tau)= & \int_{0}^{\infty} e^{-\tau u} d H(u)+\int_{-\infty}^{0} e^{-\tau u} d H(u) \\
& =1-\tau \int_{0}^{\infty} e^{-\tau u}(1-H(u)) d u+\tau \int_{-\infty}^{0} e^{-\tau u} H(u) d u
\end{aligned}
$$

откуда

$$
h(\tau)=1+h^{\prime}(0) \tau-\tau \int_{0}^{\infty}\left(e^{-\tau u}-1\right)(1-H(u)) d u+O\left(\tau^{2}\right) .
$$

Если $0<\alpha<1$, то достаточно применить к (19) лемму 1 с $g(u)=$ $e^{-u}$ и $\beta=-\alpha$. Если $1<\alpha<2$, то следует применить лемму к (20), положив $g(u)=e^{-u}-1$ и $\beta=-\alpha$. 
Если же $\alpha=1$ или $\alpha=2$, то необходимы несколько более сложные рассуждения.

Предположим, что $\alpha=1, h^{\prime}(0)=-\infty$. Из (19) легко получаем, что

$$
h(\tau)=1-\tau \int_{0}^{\infty}\left(e^{-\tau u}-\Delta(\tau u)\right) u^{-1} m(u) d u-\tau \hat{m}\left(\tau^{-1}\right)+O(\tau)
$$

где

$$
\Delta(u)= \begin{cases}1, & \text { если } 0<u \leqslant 1, \\ 0 & \text { в остальных случаях. }\end{cases}
$$

Остается положить в лемме $1 g(u)=e^{-u}-\Delta(u), \beta=-1$.

Если $\alpha=1$, но $h^{\prime}(0)>-\infty$, то из $(20)$ имеем

$h(\tau)=1+h^{\prime}(0) \tau-\tau \int_{0}^{\infty}\left(e^{-\tau u}-\Delta(\tau u)\right) u^{-1} m(u) d u+\tau \widetilde{m}\left(\tau^{-1}\right)+O\left(\tau^{2}\right)$.

Пусть $\alpha=2$. Из (20) получаем, что

$$
\begin{aligned}
h(\tau)= & 1+h^{\prime}(0) \tau-\tau \cdot \int_{0}^{\infty}\left(e^{-\tau u}-1+\tau u \Delta(\tau u)\right) u^{-2} m(u) d u \\
& +\tau^{2} \widehat{m}\left(\tau^{-1}\right)+O\left(\tau^{2}\right) .
\end{aligned}
$$

Остается положить в лемме 1

$$
g(u)=u^{-2}\left(e^{-u}-1+\Delta(u)\right), \quad \beta=0 .
$$

Лемма доказана.

Заметим, что в каждом из рассмотренных случаев мы могли бы указать вид константы $c$, которая связывает $l(u)$ и $m(u)$, использовав для этого формулы 8.212.1, 8.211.1, 3.351.5 и 3.434.1 из [17].

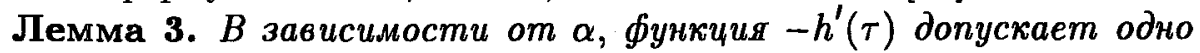
из следующих представлении:

$$
-h^{\prime}(\tau)=\left\{\begin{array}{lll}
\tau^{\alpha-1} l\left(\tau^{-1}\right), & \text { если } & 0<\alpha<1, \\
\widehat{m}\left(\tau^{-1}\right)-l\left(\tau^{-1}\right), & \text { если } & \alpha=1, h^{\prime}(0)=-\infty \\
-h^{\prime}(0)-\tilde{m}\left(\tau^{-1}\right)-l\left(\tau^{-1}\right), & \text { если } & \alpha=1, h^{\prime}(0)>-\infty \\
-h^{\prime}(0)-\tau^{\alpha-1} l\left(\tau^{-1}\right), & \text { если } 1<\alpha<2, \\
-h^{\prime}(0)-2 \tau \widehat{m}\left(\tau^{-1}\right)-\tau l\left(\tau^{-1}\right), & \text { еслu } \alpha=2 .
\end{array}\right.
$$

Здесь $\widehat{m}(x)$ и $\tilde{m}(x)$ имеют тот же смысл, что и в лемме 2.

Д о к а з а т е л ь с т в о. В этом случае, вместо (19) и (20) следует начинать с соотношений

$$
-h^{\prime}(\tau)=\int_{0}^{\infty} u e^{-\tau u} d H(u)+O(1)=\int_{0}^{\infty} e^{-\tau u}(1-\tau u)(1-H(u)) d u+O(1)
$$


и

$$
\begin{aligned}
-h^{\prime}(\tau) & =-h^{\prime}(0)+\int_{0}^{\infty} u\left(e^{-\tau u}-1\right) d H(u)+O(\tau) \\
& =h^{\prime}(0)+\int_{0}^{\infty}\left(e^{-\tau u}(1-\tau u)-1\right)(1-H(u)) d u+O(\tau)
\end{aligned}
$$

в зависимости от того, сушествует $h^{\prime}(0)$ или нет. Остальное очевидно. Лемма доказана.

Лемма 4. Вторая производная функции $h(\tau)$ допускает представления

$$
h^{\prime \prime}(\tau)=\left\{\begin{array}{lll}
\tau^{\alpha-2} l\left(\tau^{-1}\right), & \text { ecлu } & 0<\alpha<2 \\
2 \widehat{m}\left(\tau^{-1}\right)-l\left(\tau^{-1}\right), & \text { если } & \alpha=2
\end{array}\right.
$$

Д о к а 3 а т е л в с т в о нужно начинать с формулы

$$
h^{\prime \prime}(\tau)=2 \int_{0}^{\infty} u^{1-\alpha} m(u) e^{-\tau u} d u-\tau \int_{0}^{\infty} u^{2-\alpha} m(u) e^{-\tau u} d u+O(1) .
$$

Остальное очевидно.

Лемма 5. Для пюбого $k \geqslant 3$

$$
(-1)^{k} h^{(k)}(\tau)=\tau^{\alpha-k} l\left(\tau^{-1}\right)
$$

Д о к а з а т л в с т в о следует начать с формулы $(-1)^{k} h^{(k)}(\tau)=k \int_{0}^{\infty} u^{k-\alpha-1} m(u) e^{-\tau u} d u-\tau \int_{0}^{\infty} u^{k-\alpha} m(u) e^{-\tau u} d u+O(1)$

Теперь утверждение будет вытекать из леммы 1.

Нам понадобятся свойства $h(\tau-i t) / h(\tau)$ при малых $|t|$. Рассмотрим

$$
\lambda(t, \tau)=t \int_{0}^{\infty} u^{-\alpha} e^{-\tau u} \sin t u d u+\tau \int_{0}^{\infty} u^{-\alpha} e^{-\tau u}(\cos t u-1) d u
$$

где $t>0, \tau>0,0<\alpha<2$.

Если $0<\alpha<1$, то с помощью формул 3.944 .5 и 3.944 .6 из [17] и очевидных вычислений получаем

$$
\lambda(t, \tau)=c\left(t^{2}+\tau^{2}\right)^{\alpha / 2}\left(\cos \left(\alpha \operatorname{arctg} \frac{t}{\tau}\right)-\cos ^{\alpha}\left(\operatorname{arctg} \frac{t}{\tau}\right)\right) .
$$

Если $0<\alpha<1$, то $\cos \alpha u-(\cos u)^{\alpha}$ строго возрастает в интервале $(0, \pi / 2)$. Следовательно, для $t \geqslant \tau \Delta$

$$
\lambda(t, \tau) \geqslant c(\Delta) t^{\alpha}
$$

где

$$
c(\Delta)=c\left(\cos (\alpha \operatorname{arctg} \Delta)-\cos ^{\alpha}(\operatorname{arctg} \Delta)\right) .
$$


Если $1<\alpha<2$, то формулы 3.944 .5 и 3.945 .2 [17] приводят к соотношению

$$
\lambda(t, \tau)=c\left(t^{2}+\tau^{2}\right)^{\alpha / 2}\left(\cos ^{\alpha}\left(\operatorname{arctg} \frac{t}{\tau}\right)-\cos \left(\alpha \operatorname{arctg} \frac{t}{\tau}\right)\right) .
$$

Здесь $(\cos u)^{\alpha}-\cos \alpha u$ строго возрастает в интервале $(0, \pi / 2)$. Следовательно, для $t \geqslant \tau \Delta$ справедливо (21) с

$$
c(\Delta)=c\left(\cos ^{\alpha}(\operatorname{arctg} \Delta)-\cos (\alpha \operatorname{arctg} \Delta)\right) .
$$

Если же $\alpha=1$, то из формул 3.941 и 3.943 [17] следует, что

$$
\lambda(t, \tau)=t\left(\operatorname{arctg} \frac{t}{\tau}-\frac{\tau}{2 t} \ln \left(1+\frac{t^{2}}{\tau^{2}}\right)\right) .
$$

Легко видеть, что соотношение (21) сохраняет силу при

$$
c(\Delta)=\operatorname{arctg} \Delta-\frac{1}{2 \Delta} \ln \left(1+\Delta^{2}\right) .
$$

Лемма 6. Для пюбого $\Delta>0$ существует такое значение $\eta=$ $\eta(\Delta)$, ито прu $\Delta \tau \leqslant|t| \leqslant \eta$

$$
h(\tau)-\operatorname{Re} h(\tau-i t) \geqslant \begin{cases}\frac{c(\Delta)}{2}|t|^{\alpha} m\left(\frac{1}{|t|}\right), & \text { ecлu } 0<\alpha<2, \\ \frac{1}{4} e^{-\Delta^{-1}} t^{2} \hat{m}\left(\frac{1}{|t|}\right), & \text { eсли } \alpha=2 .\end{cases}
$$

Здесь $c(\Delta)$ mо же, ито $u$ в (21).

Д ок а з а т е ль с т в о. $1^{\circ}$. Пусть $0<\alpha<2$. Интегрируя по частям, получим, что

$$
\begin{aligned}
& h(\tau)-\operatorname{Re} h(\tau-i t)=\int_{0}^{\infty} e^{-\tau u}(1-\cos (t u)) d H(u)+O\left(t^{2}\right) \\
& =|t| \int_{0}^{\infty}(1-H(u)) e^{-\tau u}\left(\sin |t| u+\frac{\tau}{|t|}(\cos t u-1)\right) d u+O\left(t^{2}\right) .
\end{aligned}
$$

Для удобства будем писать $t$ вместо $|t|$. Положим

$$
J=\int_{0}^{\infty}(1-H(u)) e^{-\tau u} v(t u, z) d u
$$

где

$$
v(u, z)=\sin u+z(\cos u-1), \quad z=\frac{\tau}{t} .
$$

Представим $J$ следуюшим образом:

$$
J=\int_{0}^{X}+\int_{X}^{\epsilon / t}+\int_{\epsilon / t}^{1 /(\epsilon t)}+\int_{1 /(\epsilon t)}^{\infty}=J_{1}+J_{2}+J_{3}+J_{4} .
$$


Напомним, что для $\min (x, y)>X$ выполняется (18). Без потери общности можно выбрать $X>1$. Очевидно, что

$$
\left|J_{1}\right| \leqslant c X^{3}\left(t+z t^{2}\right) \leqslant c t .
$$

Из (18), (23) и (24) следует, что

$$
\begin{aligned}
\left|J_{2}\right| & \leqslant c t \int_{X}^{\epsilon / t} u^{-\alpha+1} m(u) d u \leqslant c t^{\alpha-1} m\left(t^{-1}\right) \int_{X t}^{\epsilon} u^{-\alpha+1} \frac{m\left(u t^{-1}\right)}{m\left(t^{-1}\right)} d u \\
& \leqslant c t^{\alpha-1} m\left(t^{-1}\right) \int_{0}^{\epsilon} u^{-\alpha+1-\delta} d u=\omega\left(\epsilon^{-1}\right) t^{\alpha-1} m\left(t^{-1}\right)
\end{aligned}
$$

при условии, что $0<\delta<2-\alpha$.

Легко видеть, что

$$
\begin{aligned}
J_{3}= & t^{\alpha-1} m\left(t^{-1}\right) \int_{\epsilon}^{1 / \epsilon} u^{-\alpha} e^{-z u} v(u, z) d u \\
& +\theta c(\epsilon) t^{\alpha-1} m\left(t^{-1}\right) \sup _{\epsilon \leqslant u \leqslant 1 / \epsilon}\left|\frac{m\left(u t^{-1}\right)}{m\left(t^{-1}\right)}-1\right| .
\end{aligned}
$$

Представим $J_{4}$ в виде

$$
\begin{aligned}
J_{4}= & \int_{1 /(\epsilon t)}^{\infty}(1-H(u)) e^{-\tau u}(\sin t u+z \cos t u) d u \\
& -z \int_{1 /(\epsilon t)}^{\infty}(1-H(u)) e^{-\tau u} d u=J_{41}+J_{42} .
\end{aligned}
$$

Так как функция $(1-H(u)) e^{-\tau u}$ монотонна, то можно применить вторую теорему о среднем значении. $\mathrm{C}$ ее помощью получаем

$$
\begin{aligned}
J_{41}= & \theta\left(1-H\left((\epsilon t)^{-1}\right)\right) \\
& \times\left(\sup _{A>(\epsilon t)^{-1}}\left|\int_{(\epsilon t)^{-1}}^{A} \sin t u d u\right|+z\left(\sup _{B>(\epsilon t)^{-1}}\left|\int_{(\epsilon t)^{-1}}^{B} \cos t u d u\right|\right)\right. \\
= & \theta \omega\left(\epsilon^{-1}\right) t^{\alpha-1} m\left(t^{-1}\right) .
\end{aligned}
$$

Подобным же образом

$$
\left|J_{42}\right| \leqslant t^{-1}\left(1-H\left((\epsilon t)^{-1}\right)\right)=\theta \omega\left(\epsilon^{-1}\right) t^{\alpha-1} m\left(t^{-1}\right) .
$$

Следовательно,

$$
J_{4}=\theta \omega\left(\epsilon^{-1}\right) t^{\alpha-1} m\left(t^{-1}\right) .
$$

Пусть $\delta_{1}$ произвольно мало. Можно выбрать $\epsilon$ настолько малым, чтобы, в соответствии с (26) и (28), для любого фиксированного $\Delta>0$ выполнялись соотношения:

$$
\max \left(\left|J_{2}\right|,\left|J_{4}\right|\right) \leqslant \delta_{1} t^{\alpha-1} m\left(t^{-1}\right)
$$


$\mathbf{K}$

$$
\max \left(\left|\int_{0}^{\epsilon} u^{-\alpha} e^{-z u} v(u, z) d u\right|,\left|\int_{1 / \epsilon}^{\infty} u^{-\alpha} e^{-z u} v(u, z) d u\right|\right) \leqslant \delta_{1} .
$$

Выберем $\eta$ таким образом, чтобы для $t<\eta$ последняя оценка в $(25)$ имела вид

$$
\left|J_{1}\right|<\delta_{1} t^{\alpha-1} m\left(t^{-1}\right)
$$

а в $(27)-$

$$
c(\epsilon) \sup _{\epsilon \leqslant u \leqslant 1 / \epsilon}\left|\frac{m\left(u t^{-1}\right)}{m\left(t^{-1}\right)}-1\right| \leqslant \delta_{1} .
$$

Объединяя эти опенки с (23) и (24), мы приходим к соотношению

$$
J=t^{\alpha-1} m\left(t^{-1}\right) \int_{0}^{\infty} u^{-\alpha} e^{-z u} v(u, z) d u+6 \theta \delta_{1} t^{\alpha-1} m\left(t^{-1}\right),
$$

справедливому для $\Delta \tau \leqslant t \leqslant \eta$. Учитывая (22) и (23), получим

$$
h(\tau)-\operatorname{Re} h(\tau-i t)=m\left(t^{-1}\right) \lambda(t, \tau)+6 \delta_{1} \theta t^{\alpha} m\left(t^{-1}\right)+O\left(t^{2}\right) .
$$

Остается вспомнить про неравенство (21) и принять во внимание произвольность $\delta_{1}$.

$2^{\circ}$. Пусть $\alpha=2$. Рассмотрим интегралы в (25) по-отдельности. Используя те же рассуждения что и в п. $1^{\circ}$, легко получаем

$$
\int_{0}^{\infty}(1-H(u)) e^{-\tau u}(1-\cos t u) d u \leqslant t m\left(t^{-1}\right) .
$$

Далее (ср. с п. $5^{\circ}$ в доказательстве леммы 2)

$$
\begin{aligned}
\int_{0}^{\infty}(\mathbb{1}-H(u)) e^{-\tau u} \sin t u d u & =\int_{0}^{\infty} u^{-2} m(u) e^{-\tau u}\left(\sin t u-\Delta_{1}(t u)\right) d u \\
+t \int_{0}^{1 / t} u^{-1} m(u) e^{-\tau u} d u & =A_{1}+t A_{2}
\end{aligned}
$$

С помощью леммы 1 получаем

$$
A_{1}=c \theta t m\left(t^{-1}\right)
$$

Для $\tau \leqslant \Delta t$ и всех достаточно малых $t$

$$
A_{2} \geqslant e^{-\Delta^{-1}} \widehat{m}\left(t^{-1}\right) \text {. }
$$

Из (16) и (30)-(32) следует, что для любого $\Delta$ и всех достаточно малых $t$

$$
\int_{0}^{\infty}(1-H(u)) e^{-\tau u} \sin t u d u \geqslant \frac{1}{2} e^{-\Delta^{-1}} \widehat{m}\left(t^{-1}\right) .
$$

Остается объединить (22), (29) и (33). Лемма доказана. 


\section{5. Свойства корня основного уравнения}

Основным мы называем уравнение $n M(s)=x$ или, иначе, $n M(\lambda-\tau)=x$. Если $x \leqslant x_{n}$, то $\tau \geqslant \tau_{n}$, где $\tau_{n}-$ корень уравнения

$$
\frac{x_{n}}{n}=M\left(\lambda-\tau_{n}\right)=-\frac{h^{\prime}\left(\tau_{n}\right)}{h\left(\tau_{n}\right)} .
$$

Пусть $a_{n}, b_{n}$ и $x_{n}$ определены как в (10)-(12).

Лемма 7. Если $\tau_{n}$ является корнем уравнения (34), то

$$
b_{n} \tau_{n} \rightarrow \infty
$$

Доказате ль с т в. Как и в лемме 1 приходится различать пять случаев.

$1^{\circ}$. Пусть $0<\alpha<1$. Из лемм 2,3 и (34) получаем $x_{n}=$ $c n \tau_{n}^{\alpha-1} l\left(\tau_{n}^{-1}\right)$. Согласно (9) и (10) имеем $n b_{n}^{-\alpha} l\left(b_{n}\right) \geqslant c$. Принимая во внимание (12), находим, что $\left(b_{n} \tau_{n}\right)^{1-\alpha} l\left(b_{n}\right) / l\left(\tau_{n}^{-1}\right) \geqslant c / \epsilon_{n}$. Так как $b_{n} \rightarrow \infty$, $\tau_{n} \rightarrow 0$, то из (18) следует, что для любого $\delta>0$

$$
\max \left(\left(b_{n} \tau_{n}\right)^{1-\delta-\alpha},\left(b_{n} \tau_{n}\right)^{1+\delta-\alpha}\right) \geqslant c \epsilon_{n}^{-1} .
$$

Таким образом, утверждение (35) доказано.

$2^{\circ}$. Пусть $\alpha=1, h^{\prime}(0)=-\infty$. Заметим, что согласно (1)

$$
a_{n}=n \int_{0}^{b_{n}} u d H(u)+n \int_{-\infty}^{0} u d H(u)+o(1)
$$

Интегрируя по частям, получим, что

$$
a_{n}=n \widehat{m}\left(b_{n}\right)-n m\left(b_{n}\right)-n c+o(1) .
$$

Комбинируя (12), (34) с леммами 2 и 3 , получаем

$$
n^{-1} x_{n}=\widehat{m}\left(b_{n}\right)-m\left(b_{n}\right)-c-z_{n} n^{-1} b_{n}(1+o(1))=\widehat{m}\left(\tau_{n}^{-1}\right)-l\left(\tau_{n}^{-1}\right) .
$$

Если $b_{n_{k}} \tau_{n_{k}} \longrightarrow 0$, то для $n=n_{k}$, в соответствии с (18),

$$
\begin{aligned}
\widehat{m}\left(\tau_{n}^{-1}\right)-\widehat{m}\left(b_{n}\right) & =\int_{b_{n} \tau_{n}}^{1} u^{-1} m\left(u \tau_{n}^{-1}\right) d u \geqslant \frac{1}{2} m\left(\tau_{n}^{-1}\right) \int_{b_{n} \tau_{n}}^{1} u^{\delta-1} d u \\
& \geqslant \frac{1}{2 \delta} m\left(\tau_{n}^{-1}\right) .
\end{aligned}
$$

Следовательно, $-c \geqslant \widehat{m}\left(\tau_{n}^{-1}\right)-\widehat{m}\left(b_{n}\right)-l\left(\tau_{n}^{-1}\right) \geqslant c m\left(\tau_{n}^{-1}\right)$, т.е.,

$$
\lim \inf _{n \rightarrow \infty} b_{n} \tau_{n}>0 \text {. }
$$


Если $b_{n_{k}} \tau_{n_{k}}<c$, то $b_{n} \tau_{n} \asymp 1$ для $n=n_{k}$ и, следовательно, $l\left(\tau_{n}\right) \sim l\left(b_{n}\right)$. Далее,

$$
\widehat{m}\left(b_{n}\right)-\widehat{m}\left(\tau_{n}^{-1}\right)=l\left(b_{n}\right) \int_{b_{n} \tau_{n}}^{1} u^{-1} d u=O\left(m\left(b_{n}\right)\right),
$$

откуда, учитывая (36),

$$
c+z_{n} n^{-1} b_{n}(1+o(1))=O\left(m\left(b_{n}\right)\right) .
$$

Из (10) следует, что $m\left(b_{n}\right)=o\left(z_{n} n^{-1} b_{n}\right)$ и, значит, (37) не выполняется. Таким образом, $b_{n} \tau_{n} \rightarrow \infty$.

$3^{\circ}$. Пусть $\alpha=1, h^{\prime}(0)>-\infty$. В этом случае

$$
a_{n}=n M-n \int_{b_{n}}^{\infty} u d H(u)+o(1) .
$$

Интегрируя по частям, получим, что

$$
a_{n}=n\left(M-\tilde{m}\left(b_{n}\right)-m\left(b_{n}\right)\right)+o(1) .
$$

Вместо (36) имеем

$n^{-1} x_{n}=M-\widetilde{m}\left(b_{n}\right)-m\left(b_{n}\right)-z_{n} n^{-1} b_{n}(1+o(1))=M-\widetilde{m}\left(\tau_{n}^{-1}\right)-l\left(\tau_{n}^{-1}\right)$.

Остается повторить только что использованные рассуждения.

$4^{\circ}$. Пусть $1<\alpha<2$. Принимая во внимание леммы 1 и 2 , уравнение (34) можно переписать следующим образом:

$$
n^{-1} x_{n}=M-c \tau_{n}^{\alpha-1} l\left(\tau_{n}^{-1}\right),
$$

откуда

$$
z_{n} b_{n}=n c \tau_{n}^{\alpha-1} l\left(\tau_{n}^{-1}\right)
$$

Принимая во внимание (9) и (10),

$$
\left(b_{n} \tau_{n}\right)^{\alpha-1} \frac{l\left(\tau_{n}^{-1}\right)}{l\left(b_{n}\right)} \geqslant c z_{n}
$$

Остается, как и в случае $0<\alpha<1$, применить (18).

$5^{\circ}$. Наконец, пусть $\alpha=2$. Из (10), (34) и лемм 2,3 следует, что

$$
n^{-1} z_{n}=M-2 \tau_{n} \hat{m}\left(\tau_{n}^{-1}\right)(1+o(1))=M-z_{n} n^{-1} b_{n} .
$$

Интегрируя по частям, получим, что

$$
\int_{-x}^{x} u^{2} d H(u)=\int_{0}^{x} u^{2} d H(u)+O(1)=2 \widehat{m}(x)(1+o(1)) .
$$


Из (10) следует, что $n b_{n}^{-2} \widehat{m}\left(b_{n}\right) \geqslant c$. Учитывая (38),

$$
z_{n} n^{-1} b_{n} \leqslant \tau_{n} \widehat{m}\left(\tau_{n}^{-1}\right)
$$

откуда

$$
\left(b_{n} \tau_{n}\right)^{\alpha-1} \frac{\widehat{m}\left(\tau_{n}^{-1}\right)}{\widehat{m}\left(b_{n}\right)} \geqslant c z_{n} .
$$

Остается, как и в случае $0<\alpha<1$, воспользоваться неравенствами (18). Лемма доказана.

\section{6. Доказательство теоремы 2}

Сначала мы докажем ряд соотношений, играюших важную роль в доказательстве теоремы.

С помощью лемм 4 и 7, а также соотношений (18) и (10), получаем

$$
n \tau^{2} h^{\prime \prime}(\tau)=c n \tau^{2} l_{1}\left(\tau^{-1}\right)=c n b_{n}^{-\alpha} l_{1}\left(b_{n}\right)\left(b_{n} \tau\right)^{\alpha} \frac{l_{1}\left(\tau^{-1}\right)}{l_{1}\left(b_{n}\right)} \geqslant c\left(b_{n} \tau\right)^{\alpha-\delta}
$$

где

$$
l_{1}(x)= \begin{cases}l(x), & \text { если } 0<\alpha<2 \\ \widehat{m}(x), & \text { если } \alpha=2\end{cases}
$$

Таким образом,

$$
n \inf _{\tau_{n} \leqslant \tau \leqslant \delta} \tau^{2} h^{\prime \prime}(\tau) \longrightarrow \infty
$$

Далее

$$
n h^{\prime \prime}(\tau)=n c b_{n}^{-\alpha} l_{1}\left(b_{n}\right)\left(b_{n} \tau\right)^{\alpha-2} b_{n}^{2} \frac{l_{1}\left(\tau^{-1}\right)}{l_{1}\left(b_{n}\right)} .
$$

Если $0<\alpha<2$, то, как и прежде, для $\tau \geqslant \tau_{n}$ получаем, что

$$
n b_{n}^{-2} h^{\prime \prime}(\tau) \leqslant c\left(b_{n} \tau\right)^{\alpha-2+\delta}=o(1) .
$$

Если же $\alpha=2$, то цля $\tau \geqslant \tau_{n}$

$$
\frac{l_{1}\left(\tau^{-1}\right)}{l_{1}\left(b_{n}\right)}=\frac{\widehat{m}\left(\tau^{-1}\right)}{\widehat{m}\left(b_{n}\right)} \leqslant c
$$

поскольку $\widehat{m}(x)$ монотонно возрастает. Следовательно, $n b_{n}^{-2} h^{\prime \prime}(\tau) \leqslant c$. Таким образом,

$$
n b_{n}^{-2} \sup _{\tau_{n} \leqslant \tau \leqslant \delta} h^{\prime \prime}(\tau)= \begin{cases}\theta \omega(n), & \text { если } 0<\alpha<2 \\ \theta c, & \text { если } \alpha=2\end{cases}
$$

Положим

$$
\rho(\tau)=\left|h^{\prime \prime \prime}(\tau)\right|\left(h^{\prime \prime}(\tau)\right)^{-3 / 2}
$$


Из лемм 4 и 5 следует, что

$$
\rho(\tau)= \begin{cases}c \tau^{-\alpha / 2}\left(l\left(\tau^{-1}\right)\right)^{-1 / 2}, & \text { если } 0<\alpha<2, \\ c \tau^{-1}\left(\widehat{m}\left(\tau^{-1}\right)\right)^{-3 / 2} l\left(\tau^{-1}\right), & \text { если } \alpha=2,\end{cases}
$$

и, следовательно,

$$
\tau \sqrt{h^{\prime \prime}(\tau)}= \begin{cases}O\left((\rho(\tau))^{-1}\right), & \text { если } 0<\alpha<2, \\ o\left((\rho(\tau))^{-1}\right), & \text { если } \alpha=2 .\end{cases}
$$

Перейдем непосредственно к доказательству теоремы. Предположим сначала, что $x \leqslant x_{n}$. Очевидно можно ограничиться случаем $n M_{\delta} \leqslant x \leqslant x_{n}$.

Пусть $\xi_{j}(s), j=1,2, \ldots$, независимы и имеют общее распределение $(13)$, а $\zeta_{n}(s)=\xi_{1}(s)+\cdots+\xi_{n}(s)$. Определим на борелевских множествах мepy

$$
Q_{n s}(A)=\sqrt{2 \pi n M^{\prime}(s)} \mathbf{P}\left\{\zeta_{n}(s)-n M(s) \in A\right\} .
$$

Прежде всего покажем, что $Q_{n s}$ слабо сходится при $n \rightarrow \infty$ к мере Лебега, причем равномерно по $s, 0<\delta \leqslant s \leqslant \lambda-\tau_{n}$. Тут нужно слегка изменить форму классической интервальной локальной предельной теоремы, приведенной в [15] (см. также [18]). Положим

$$
q(t)=e^{-i t M(s)} \mathrm{E} e^{i t \xi_{1}(s)}=e^{-i t M(s)} \frac{h(\tau-i t)}{h(\tau)}, \quad s=\lambda-\tau .
$$

Введем индикаторную функцию

$$
e(u)= \begin{cases}1, & \text { если } u \in[0, y), \\ 0, & \text { если } u \notin[0, y),\end{cases}
$$

где $y>0$ фиксированно. Рассмотрим далее для произвольно малого $\varepsilon>0$ монотонные $\varepsilon$-аппроксимации функции $e(u)$, т.е. такие функции $e_{-}(u)$ и $e_{+}(u)$, что

a) $e_{-}(u) \leqslant e_{+}(u)$;

b) $\int\left(e_{+}(u)-e_{-}(u)\right) d u<\varepsilon$

c) $\widehat{e}_{ \pm}(t)=\int e^{i t u} e_{ \pm}(u) d u$ имеют компактные носители.

Из тождества Парсеваля следует, что

$$
\mathbf{E} e\left(\zeta_{n}(s)-x\right) \leqslant \mathbf{E} e_{+}\left(\zeta_{n}(s)-x\right)=\frac{1}{2 \pi} \int e^{-i t x} \widehat{e}_{+}(-t) q^{n}(t) d t
$$

Подобным же образом получаем, что

$$
\mathbf{E} e\left(\zeta_{n}(s)-x\right) \geqslant \mathbf{E} e_{-}\left(\zeta_{n}(s)-x\right)=\frac{1}{2 \pi} \int e^{-i t x} \hat{e}_{-}(-t) q^{n}(t) d t
$$


Учитывая свойство с) монотонной $\varepsilon$-аппроксимации, существует такое $T>0$, что

$$
\int e^{-i t x} \widehat{e}_{ \pm}(-t) q^{n}(t) d t=\int_{|t T|<T} e^{-i t x} \widehat{e}_{ \pm}(-t) q^{n}(t) d t
$$

Достаточно показать, что

$$
\sup _{\delta \leqslant s \leqslant \lambda-\tau_{n}}\left|\sqrt{n M^{\prime}(s)} \int_{|t|<T} \widehat{e}_{ \pm}(t) q^{n}(t) d t-\frac{\widehat{e}_{ \pm}(0)}{\sqrt{2 \pi}}\right|=o(1) .
$$

Обозначим оцениваемый интеграл в (43) через $J$. Представим его в виде суммы интетралов

$$
J=\int_{|t|<\Delta \tau}+\int_{\Delta \tau \leqslant|t| \leqslant \eta}+\int_{\eta<|t|<T}=J_{1}+J_{2}+J_{3} .
$$

Здесь величина $\eta$ та же, что и в лемме 6.

Положим

$$
\beta(s)=\frac{\mathbf{E}\left|\xi_{1}(s)-M(s)\right|^{3}}{M^{\prime}(s)^{3 / 2}}
$$

Напомним, что

$$
M^{\prime}(s)=h^{\prime \prime}(\lambda-s)=\mathbf{D} \xi(s) .
$$

Известно, что (см., например, (5.6) в гл. XVI монографии [16])

$$
n \ln q\left(\frac{t}{\sqrt{n M^{\prime}(s)}}\right)=-\frac{t^{2}}{2}+\theta n^{-1 / 2}|t|^{3}
$$

по крайней мере, для $|t|<\sqrt{n} / \beta(s)$.

Из лемм 1, 2 и (41) следует, что $\beta(s) \sim \rho(\tau)$ при $\tau=\lambda-s \longrightarrow 0$. Таким образом, согласно (42) можно выбрать $\Delta>0$ так, чтобы

$$
\tau \Delta \sqrt{M^{\prime}(s)} \leqslant \frac{1}{\beta(s)} .
$$

Из леммы 7 и соотношения (39) следует, что

$$
\inf _{\tau_{n} \leqslant \tau \leqslant \delta} n \tau^{2} M^{\prime}(\lambda-\tau) \longrightarrow \infty
$$

Объединение (44), (45) и (46) дает

$$
\sqrt{n M^{\prime}(s)} J_{1}=\frac{\widehat{e}_{ \pm}(0)}{\sqrt{2 \pi}}+o(1) .
$$


С помоцью леммы 6 получаем, что

$$
\left|J_{2}\right| \leqslant \sup _{|t| \leqslant \eta}\left|e_{ \pm}(t)\right| \int_{\Delta \tau \leqslant|t| \leqslant \eta}|q(t)|^{n} d t \leqslant c \int_{\Delta \tau}^{\eta} \exp \left(-n c t^{\alpha} l_{1}\left(t^{-1}\right)\right) d t
$$

где, как и прежде,

$$
l_{1}(x)= \begin{cases}l(x), & \text { если } 0<\alpha<2, \\ \hat{m}(x), & \text { если } \alpha=2 .\end{cases}
$$

Без потери обшности можно предположить, что $1 / \eta \geqslant X$, а $n$ достаточно велико. Тогда из (10) и (18) следует, что $n t^{\alpha} l\left(t^{-1}\right) \geqslant c\left(t b_{n}\right)^{\alpha-\delta}$. Принимая во внимание (40), получаем, что

$$
\sqrt{n M^{\prime}(s)}\left|J_{2}\right| \leqslant c b_{n}^{-1} \sqrt{n M^{\prime}(s)} \int_{\Delta \tau b_{n}}^{\infty} \exp \left(-c t^{\alpha-\delta}\right) d t=o(1) .
$$

Очевидно, что

$$
\sup _{0 \leqslant s \leqslant \lambda} \sup _{\eta \leqslant|t| \leqslant T}|q(t)|=q<1
$$

Следовательно, в соответствии с (40)

$$
\sqrt{n M^{\prime}(s)}\left|J_{3}\right| \leqslant c b_{n} q^{n}=o(1) .
$$

Теперь оценка (43) следует из (44) и (48)-(49). Далее

$$
\mathbf{P}\left\{\zeta_{n}>x\right\}=\frac{f^{n}(s) e^{-s x}}{\sqrt{2 \pi n M^{\prime}(s)}} \int_{(0, \infty)} e^{-s u} Q_{n s}(d u) .
$$

Отсюда вытекает утверждение теоремы, поскольку функции $e^{-s u}, 0 \leqslant$ $s \leqslant \lambda$, равностепенно непрерывны и равномерно интегрируемы на $(0, \infty)$.

Пусть теперь $x-a_{n} \asymp b_{n}$. Положим

$$
Q_{n \lambda}^{(x)}(A)=\mathbf{P}\left\{\zeta_{n}(\lambda)-x \in A\right\}
$$

Очевидно, что

$$
\mathbf{P}\left\{\zeta_{n}>x\right\}=f^{n}(\lambda) e^{-\lambda x} \int_{(0, \infty)} e^{-\lambda u} Q_{n \lambda}^{(x)}(d u) .
$$

Из интервальной локальной предельной теоремы (см., например, [18]) получаем, что

$$
b_{n} \int_{(0, \infty)} e^{-\lambda u} Q_{n \lambda}^{(x)}(d u)=g_{\alpha}\left(b_{n}^{-1}\left(x-a_{n}\right)\right) \int_{0}^{\infty} e^{-\lambda u} d u+o(1)
$$

равномерно по $x,-\infty<x<\infty$, откуда следует утверждение теоремы. 


\section{СПИСОК ЛИТЕРАТУРЫ}

1. Nagaev S. V. Large deviations of sums of independent variables. - Ann. Probab., 1979, № 7, p. 745-789.

2. Розовский Л. В. Вероятности больших уклонений. - Теория вероятн. и ее примен., 1993 , т. 38 , в. 1 , с. $79-109$.

3. Боровков A. A., Могульский $A$. A. Большие уклонения и проверка статистических гипотез. - Труды ин-та матем. СО РАН, 1992, № 2, с. 3-220.

4. Dembo A., Zeitouni $O$. Large deviations techniques and applications. Boston, MA: Jones and Bartlett, 1993.

5. Deuschel J. D., Strook D. W. Large Deviations. Duluth, London: Academic Press, 1989.

6. Ellis R. S. Entropy, Large Deviations and Statistical Mechanics. Berlin-New York: Springer-Verlag, 1985.

7. Крамер $Г$. Об одной новой предельной теореме теории вероятностей. - Успехи матем. наук, 1944, в. Х, с. $166-178$.

8. Нагаев $A$. В. Новые теоремы о больших уклонениях в условиях Крамера. В сб.: 4-я Международная конференция по теории вероятн. и матем. статист. Тезисы докладов, 1985, № 1, с. 32-33.

9. Петров В. В. О вероятностях больших уклонений сумм независимых случайных величин. - Теория вероятн. и ее примен., 1965, т. Х, в. 2, с. 310-322.

10. Золотарев $B . M$. Об одной новой точке зрения на предельные теоремы, учитывающие большие уклонения. - В сб.: Труды 6-го Всесоюзного совешания по теории вероятн. и матем. статист. Вильнюс: Госиздат, 1962, с. 43-47.

11. Ким Л. B., Нагаев $A . B$. О несимметричной проблеме больших уклонений. Теория вероятн. и ее примен., 1975, т. XX, в. 1, с. 58-68.

12. Нагаев А. В. Большие ј'клонения для одного класса распределений. - В сб.: Предельные теоремы теории вероятностей. Ташкент: Изд. АН УзССР, 1963, с. 55-68.

13. Нагаев А. В. Локальные предельные теоремы с учетом больших уклонений. В сб.: Предельные теоремы и вероятностные процессы. Ташкент: ФАН, 1967, c. $71-88$.

14. Ибрагимов И. А., Линкик Ю. В. Независимые и стационарно связанные величины. М.: Наука, 1965, 524 c.

15. Bretagnôlle $J$., Dacunha-Castelle $D$. Théorèmes limites à distance finie pour les marches aléatoires. - Ann. Inst. H. Poincaré, 1968, № 4, p. 25-73.

16. Феллер B. Введение в теорию вероятностей и ее применения. Том II. М.: Мир, 1984, $752 \mathrm{c}$.

17. Градитейн И. С., Рыжик И. М. Таблицы интегралов, сумм, рядов и произведений. М.: Физматгиз, 1963.

18. Нагаев $A$. В. Некоторые замечания по поводу многомерных локальных предельных теорем. - Матем. заметки, 1973, № 14, с. 559-563.

19. Höglund $T$. A unified formulation of the central limit theorem for small and large deviation from the mean. - Z. Wahrscheinlichkeitstheor. verw. Geb., 1979, B. 49, S. 105-117. 